\title{
Prolapso uretral: un diagnóstico diferencial del abuso sexual en niños.
}

\author{
Urethral prolapse: a differential diagnosis of sexual abuse in \\ children.
}

\section{GA. Montes ${ }^{1}$, HA. García ${ }^{2}$ y ME. Pataquiva ${ }^{3}$}

RESUMEN

Objetivo: Describir el caso de una paciente con prolapso uretral que consultó por sospecha de trauma genital no accidental (abuso sexual) y realizar una revisión de la literatura.

Material y métodos: Se describió el caso clínico de un paciente con prolapso uretral quien fue llevado por la autoridad para examen sexológico forense ante el Instituto Nacional de Medicina Legal y Ciencias Forenses de Bogotá - Colombia, por sospecha de abuso sexual. Posteriormente se realizó una búsqueda exhaustiva de la literatura en diferentes bases de datos con las que se sustentó la discusión del caso.

Resultados: Se presentó el caso de una paciente de 23 meses de edad con historia de sangrado vaginal con sospecha de trauma genital, abuso sexual y maltrato infantil que después de ser evaluada adecuadamente por especialistas en el área genital y con examen riguroso bajo sedación se hizo un diagnóstico de prolapso uretral y fue atendida y manejada para tal fin.

Conclusiones: El concepto de un médico especialista forense es de gran importancia dentro del proceso judicial en un delito sexual, por esta razón el examen sexológico forense requiere de una gran rigurosidad que permita llegar a un diagnóstico acertado y no deben olvidarse los diagnósticos diferenciales en estos pacientes para hacer un manejo adecuado tanto médico como judicial.

Palabras clave: prolapso uretral, uretra, prolapso, trauma, abuso sexual.

Cuad Med Forense 2009; 15(58):289-292

\section{ABSTRACT}

Purpose: To describe the case of a patient with urethral prolapse who consulted for suspected nonaccidental genital trauma (sexual abuse) and to review the literature.

Materials and methods: It was described the case of a patient with urethral prolapse who was taken by the authorities for forensic sexological examination at National Institute of Legal Medicine and Forensic Sciences in BogotaColombia on suspicion of sexual abuse. Subsequently conducted a thorough literature search in different databases that support the discussion of the case.

Results: It is presented the case of a 23-month-old girl with a history of vaginal bleeding with suspected genital trauma, sexual abuse and child abuse after being thoroughly assessed by experts in the genital area and rigorous examination under sedation was diagnosed urethral prolapse and was treated and managed for that purpose.

Conclusions: The concept of a forensic medical specialist is of great importance in the judicial process in a sexual offense, which is why forensic sexological examination requires a high standard that will lead to a correct diagnosis and should not miss the differential diagnosis in these patients to properly manage both medical and legal.

Key words: urethral prolapse, urethra, prolapse, trauma, sexual abuse.

Correspondencia: Dr. Guillermo Andrés Montes Loaiza. Calle 45 № 6-58 Apto 619. Bogotá (Colombia). Tfno.: 7584456 3173001114. E-mail: gmontex@hotmail.com.

1 Especialista en Medicina Forense. Universidad Nacional de Colombia. Bogotá (Colombia).

2 Servicio De Urología. Universidad del Valle. Cali (Colombia).

3 Residente de Medicina Forense. Universidad Nacional de Colombia. Bogotá (Colombia). 


\section{INTRODUCCIÓN:}

El examen sexológico forense en lactantes menores es un reto para el clínico y para el médico especialista forense. El objetivo de un examen médico-legal sexológico es determinar la presencia de lesiones y dar elementos suficientes para poder determinar el origen de las mismas [I], así mismo, este examen permite identificar y recolectar evidencias [2] que sirvan de apoyo en la investigación judicial para aclarar si existió o no un delito sexual, por tanto debe seguir una adecuada planeación y una técnica rigurosa para no olvidar detalle.

El relato de la menor es de gran importancia para la contextualización del caso y ayuda entonces a la planeación a la hora del examen físico y de la recolección de evidencias [3, 4]. Sin embargo, en el caso de lactantes menores, el relato puede estar limitado o ser nulo, por la corta edad del examinado, tomando gran importancia en este caso el relato de los padres o personas que rodean al menor.

Es importante siempre hacer el diagnóstico diferencial ante la presencia de hallazgos patológicos durante un examen sexológico forense, con lesiones traumáticas accidentales o lesiones de origen no traumático [5], puesto que un diagnóstico errado puede llevar a la búsqueda de un culpable inexistente e incluso la privación de la libertad de una persona que no ha cometido un delito y que puede ser condenado de forma equivocada.

El objetivo del presente caso clínico es hacer una revisión actualizada de la literatura y una puesta a punto sobre el enfoque diagnóstico, seguimiento y diagnóstico diferencial del abuso sexual, en particular con una patología de difícil diagnóstico en las lactantes menores como lo es el prolapso uretral.

\section{REPORTE DEL CASO:}

Se reporta el caso de una paciente de género femenino de 23 meses de edad que consulta a la unidad de urgencias de un hospital de tercer nivel por sangrado vaginal, con antecedente de episodio previo. No hay historia de abuso sexual o trauma genital. Al examen físico se evidenció abundante sangrado genital por lo que consideraron dentro de sus diagnósticos diferenciales: trauma, abuso sexual y maltrato infantil, por lo que el caso es reportado a la autoridad, quienes por medio de un grupo élite de manejo de delitos sexuales, deciden remitir para examen médico-legal.

Durante la anamnesis, el médico especialista forense tomó toda la información disponible y ante la información aportada por la madre, la cual recalcaba que no sospechaba de abuso sexual, prosiguió al examen genital, encontrando que la menor presentaba genitales femeninos infantiles normoconfigurados con hematoma en el meridiano de las 12 de $0.3 \times 0.3$ $\mathrm{cm}$ que no permitía ver el borde libre del himen, por lo que no fue posible descartar desgarros a éste nivel (Figura $\mathrm{n}^{\circ} \mathrm{I}$ ), se evidenció sangrado reciente en región vaginal, pañal y pantalón. No se observaron otras lesiones.

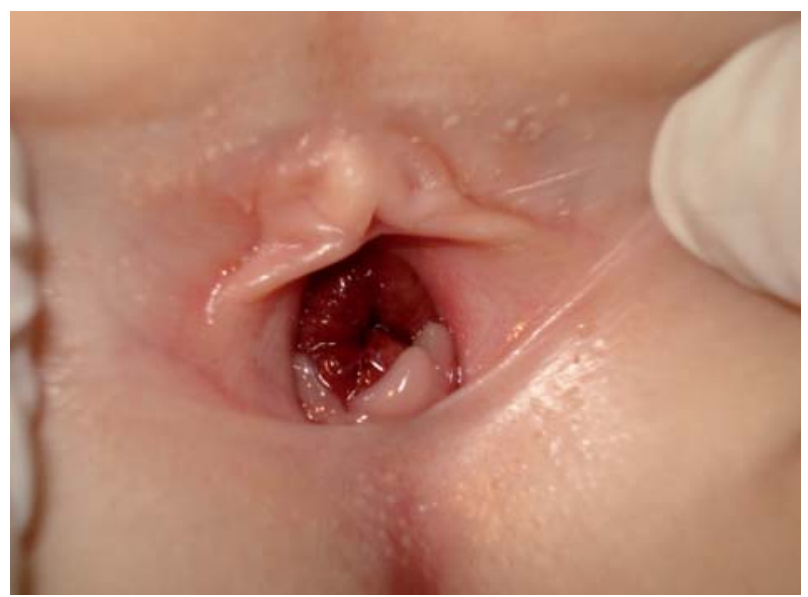

Fig. 1.- Ilustración de los genitales de una paciente con prolapso uretral. 
El grupo élite de manejo de delitos sexuales entregó al médico especialista forense muestras que fueron tomadas en el centro hospitalario donde fue inicialmente valorada la menor, muestras que ante los hallazgos al examen físico, el médico especialista forense consideró pertinente enviar al laboratorio de biología del Instituto Nacional de Medicina Legal y Ciencias Forenses para búsqueda de evidencia traza (espermatozoides y ADN) bajo cadena de custodia.

En la conclusión, el médico especialista forense asocia la lesión encontrada con trauma genital y describe que no es posible con los hallazgos determinar si es de origen accidental o no.

Posterior al examen médico-legal, la menor es remitida a un Hospital Infantil, con gran experiencia en el manejo del abuso sexual y el maltrato infantil. El personal médico de dicho Hospital solicita una nueva valoración por otro médico especialista forense, quien acude al Hospital para un nuevo examen. Dicho examen es realizado bajo sedación, utilizando lupa durante el examen genital, encontrando que la lesión descrita por el primer médico no era más que mucosa uretral prolapsada, que simulaba un hematoma en el meridiano de las 12 horas. Se hace entonces el diagnóstico de prolapso uretral. La menor es llevada a manejo quirúrgico con corrección del prolapso.

\section{DISCUSIÓN:}

El caso trata de una menor en quien se sospecha abuso sexual ante la presencia de sangrado y lesión en región genital. Posterior al examen médico-legal se conceptúa de manera errónea por parte del médico especialista forense que la menor presenta una lesión de origen traumático. La no rigurosidad durante el examen y el poco índice de sospecha no permitió al médico tener entre sus diagnóstico diferenciales el prolapso uretral.

El prolapso uretral es considerado una patología poco frecuente, a veces problemática que requiere manejo especializado. Se trata de una eversión de la mucosa uretral a través del meato [6].

Usualmente se presenta en pacientes mujeres preadolescentes afroamericanas, con pocos reportes en niñas [7], sin embargo se pueden presentar entre 14 meses a 10 años [8] y por otro lado también se pueden presentar en pacientes blancas postmenopáusicas $[6,8,9]$.

Su etiología no es clara, sin embargo la teoría más aceptada es la falla en la fijación de la musculatura perineal asociada a aumentos episódicos de la presión intraabdominal [8, 10].

Se presenta como una masa indolora, edematosa, inflamada con sangrado fácil en la mayoría de los casos [6-8], también se asocia a disuria e inhabilidad para la micción.

Al examen físico se presenta un masa vulvar anterior en "forma de dona" que engloba toda la apertura vulvar, edematosa, a veces requiere cateterismo o la visualización de la micción para identificar el meato [6, 7]. El diagnóstico es clínico, sin embargo algunas veces es necesario la utilización de ayudas diagnósticas como la cistoscopia [7].

El examen físico debe ser riguroso, con especial detalle en la región genital, sin olvidar la búsqueda de lesiones extragenitales [1 I, I2]. En el lactante menor, el pequeño tamaño de los genitales dificulta su examen, pero al tiempo aumenta la probabilidad de encontrar lesiones ante un trauma inflingido.

Ante el examen sexológico forense en una lactante menor es de gran importancia tener en cuenta los diagnósticos diferenciales probables al carecer en muchos casos de información brindada directamente por la menor en la anamnesis, entre ellos se pueden presentar: Prolapso vesical, prolapso de pólipo uretral, ureterocele, ectopia ureteral, quistes, hidrometrocolpos, condiloma acuminado, absceso, tumores y trauma genital.

La apariencia de la lesión lleva a la malinterpretación de abuso sexual [6] si no se consigue un adecuado rigor durante el examen y se sugiere que el examen genital debe ser realizado bajo 
anestesia [8]. La interpretación de sospecha de trauma genital (abuso) fue lo sucedido con la paciente que describimos y por lo cual decidimos hacer la revisión de la literatura. El examen sexológico debe incluir el uso de ayudas si es necesario, como lupa, toma de fotografías y solicitud de interconsulta con otros especialistas como urólogos o pediatras.

El tratamiento tiene un enfoque urológico, es muy controvertido, va desde el manejo médico con éxito sólo en 33\% de los casos, $[6,13,14]$ así como quirúrgico que debe ser realizado en pacientes con trombosis, sangrado, necrosis, persistencia del prolapso a pesar de manejo médico o recurrencia del mismo [6].

\section{CONCLUSIONES:}

El concepto pericial brindado por un médico especialista forense es de gran importancia dentro del proceso judicial de investigación de un delito sexual. Por esta razón, el examen sexológico forense requiere de una gran rigurosidad que permita llegar a un diagnóstico acertado y a la toma adecuada de evidencia que permita llevar en un adecuado curso una investigación judicial. Para ello el médico forense debe siempre tener presente diagnósticos diferenciales ante la presencia de lesiones que pueden ser confundidas con trauma, en este caso particular, el prolapso uretral, un diagnóstico diferencial que debe ser conocido por el médico forense para identificarlo cuando se presenta.

\section{BIBLIOGRAFÍA:}

I.Herrman B, Crawford J. Genital Injuries in Prepubertad Girls From Inline Skating Accidents. Pediatrics 2002; 110:16.

2.Mora R, Sánchez M. La evidencia física y la cadena de custodia en el procedimiento acusatorio. I ed. Bogotá: Editores Gráficos Colombia Ltda. 2007.

3.Merrit D. Vulvar and genital trauma in paediatric and adolescent gynecology. Current Opinion in Obstetrics and Gynecology 2004; 16:371-381.

4.Caffo E, Belaise C. Psychological aspects of traumatic injury in children and adolescents. Child Adolesc Psychiatr Clin North Am. 2003; 12:493-535

5.Heppenstall-Heger A, McConnell G, Ticson L, Guerra L, Lister J, Zaragoza T. Healing Patterns in anogenital injuries: A longitudinal Study of Injuries Associated with Sexual Abuse, Accidental Injuries, or genital surgery in the preadolescent child. Pediatrics. 2003; II2:829-837

6. Shetty A., Coffman K., Harmon E. Urethral prolapse. J Pediatr 1998; 133: 552.

7. Rudin JE., Geldt VG., Aleecsev EB. Prolapse of urethral mucosa in white female children: experience with 58 cases. Journal of pediatric surgery. 1997; 32(3): $423-425$.

8. Valerie E., Gilchrist F., Frischer J., Scriven R., Klotz DH., Ramenofsky ML. Diagnosis and treatment of urethral prolapsed in children. Urology. 1999; 54: 1082 - 1084

9. Jerkins $G$, Verheeck $K$, and Noe HN. Treatment of girls with urethral prolapse. J Urol 132: 733-734, 1994.

I0. Lowe F, Hill G, Jeffs R. Urethral prolapse in children: insights into etiology and management. J Urol 1986; 135: 100 - 103.

II. Agudelo M. Guía Práctica para el Dictamen Sexológico Forense. Instituto Nacional de Medicina Legal y Ciencias Forenses. Bogotá. 2001.

12. Suárez M.L., González F.J. Importancia de la exploración médicoforense en las agresiones sexuales a menores. Cuad. Med. Forense. 2003; 31:37-45

13. Trotman M., Brewster E. Prolapse of the urethral mucosa in prepubertal west indians girls. Br J Urol. 1993; 73: 503- 506.

14. Ho KL., Chu SM., Tam PC. Reduction of strangulated urethral prolapsed using local anesthesia with hyaluronidase. J Urol. 2003; 169:288 